\title{
Méthodologie et outil de diagnostic 4.0 : définir sa stratégie de transition 4.0 pour le management de la chaîne logistique
}

\author{
loana Deniaud (Da, François Marmier (Da,b et Jean-Louis Michalak ${ }^{a}$ \\ aBeta, CNRS UMR 7522, Université de Strasbourg, Strasbourg, France; 'blMT Mines Albi, Université de Toulouse, Toulouse, France
}

\begin{abstract}
RÉSUMÉ
Les donneurs d'ordre expriment des exigences de performance envers les entreprises participantes à leur chaîne logistique. Pour obtenir de meilleures performances au niveau global, la transition vers le 4.0 doit être faite avec l'ensemble de parties prenantes. Pour cadrer cette transformation l'article vise à définir une stratégie adaptée aux moyens de chaque maillon afin d'optimiser globalement la chaîne. Les contributions, basées sur une recherche déductive, sont : (1) une méthodologie de définition d'une stratégie pertinente vers une transformation 4.0 d'une chaîne logistique ; (2) un modèle d'évaluation qualitative du niveau de maturité des entreprises et de l'ensemble de leur chaîne logistique, sur les différents axes caractérisant la transformation 4.0 ; (3) sur la base de l'écart constaté dans chaque maillon par rapport aux exigences des donneurs d'ordre, nous proposons un outil d'aide à la décision permettant de déterminer et prioriser la stratégie de développement à adopter pour une transformation vers la chaîne logistique 4.0 .
\end{abstract}

\section{Methodology and diagnostic tool 4.0: defining the 4.0 transition strategy for supply chain management}

\begin{abstract}
Clients and supply chain managers express performance requirements to companies participating in their supply chain. To ensure an overall improvement in supply chain performance, the transition to 4.0 must encompass all of the involved stakeholders. With a view to facilitating the determination of the transformations required of companies, this article sets out to identify a strategy for optimising the overall supply chain in a manner consistent with each stakeholder's resources. Based on deductive research, we propose the following: (1) a methodology for defining a relevant strategy for 4.0 transition of a supply chain; (2) a qualitative model for assessing the maturity of companies and their entire supply chain, in various areas characterising the 4.0 transformation; and (3) a decision-making tool, taking into consideration each stakeholder's deviation from clients' requirements, with a view to identifying and prioritising the development strategy to be followed for a transition to a 4.0 supply chain.
\end{abstract}

\author{
MOTS CLÉS \\ Transformation 4.0 ; chaîne \\ logistique ; évaluation : \\ modèle de maturité

\section{KEYWORDS} \\ 4.0 transformation; supply \\ chain; assessment; maturity \\ model
}

\section{Introduction}

La concurrence est toujours plus exacerbée et les clients de plus en plus exigeants en terme de personnalisation de produits et services, à l'affut des nouvelles innovations, tout en gardant leurs exigences en terme de "coûts, qualité et délais » qu'ils avaient pour des produits fabriqués en masse (Christopher et Towill 2000). Le grand défi de l'entreprise d'aujourd'hui est de développer des stratégies pour s'adapter continuellement à ces nouvelles exigences du marché, en devenant plus " intelligente ", plus transparente, plus flexible et plus agile à tous les niveaux (Brown et Bessant 2003 ; Prinz et al. 2016 ; Barreto, Amaral, et Pereira 2017). Au risque de leur survie, les entreprises doivent donc combiner les avantages des modèles artisanaux, fabriquant des produits personnalisés et des modelés industriels basés sur la production de masse. On en conclut que les objectifs sont multiples et parfois contradictoires : réduire les coûts et les délais et augmenter la capacité à innover et à proposer des produits et services personnalisés, répondant à des exigences accrues des clients.

Ces dernières années, de nouveaux concepts d'organisation se sont développés dans un rythme soutenu afin de répondre, en partie, à ces nombreux défis : l'entreprise ambidextre (capable de développer simultanément des projets innovants et routiniers ; O'Reilly et Tushman 2013), l'entreprise Lean (qui part à la chasse aux gaspillages pour réduire les coûts, augmenter la réactivité et le niveau de performance ; Womack et Jones 1997), l'entreprise agile basée sur une décentralisation de la prise de décisions et l'apprentissage organisationnel (Alavi et al. 2014) e $t$ 
l'entreprise durable qui respecte l'environnement (Elkington 1998). Plus récemment, le concept d'entreprise 4.0 (Kagermann, Wahlster, et Helbig 2013) tire parti des concepts précédents en profitant de la modernisation des outils industriels par le numérique (internet des objets, big data, intelligence artificielle, systèmes cyber-physique ; Hermann et al. 2016 ; Lee, Bagheri, et Kao 2015 ; Hofmann et Rüsch 2017). L'un de principaux défis de l'industrie du futur est de faire le lien entre le monde digital et le système physique par le développement de systèmes cyber-physiques où les machines et la matière sont équipées de capteurs qui coopèrent ensemble à travers un réseau afin de s'autooptimiser (Lee, Bagheri, et Kao 2015 ; Herberer, Lau, et Behrendt 2017). Pour cela les entreprises doivent innover continuellement. Selon I'Institut national de la statistique et des études économiques (INSEE, 2018), la moitié des sociétés innovent et plus de $15 \%$ innovent dans la chaîne logistique, notamment par l'introduction de systèmes de gestion informatisés. En effet, l'évolution technologique et les outils informatiques permettent une collaboration en temps réel entre les différents services ou processus et produits intelligents, le partage d'information et un suivi en temps réel de la performance des processus. Pour optimiser les flux de produits personnalisés, amplifier l'innovation des produits/services et améliorer la gestion des ressources, les systèmes d'information permettent des connexions à tous les niveaux : des fournisseurs des fournisseurs aux clients des clients en passant par les équipements. II ne suffit donc pas qu'une entreprise opère seule, en interne, une transition vers le 4.0 , mais il est nécessaire que l'ensemble de la chaîne logistique progresse dans cette transition dans une logique d'entreprise étendue. II s'agit d'écouter et de comprendre les exigences du donneur d'ordre dans la chaîne logistique globale, transformer ces exigences en besoin interne pour chaque maillon en y associant des spécifications mesurables, et enfin de définir des stratégies tangibles, pertinentes en fonction de ses propres moyens.

La plupart des entreprises de la chaîne logistique ne savent pas comment réaliser cette transition vers le 4.0. Pour cela, nous soutenons l'idée qu'elles doivent être en mesure de réaliser un diagnostic factuel de leur fonctionnement au regard des différents axes de la transformation 4.0. Elles ne savent pas non plus comment fixer des objectifs atteignables définissant une stratégie de transformation vers le 4.0. En effet, il peut être difficile pour une entreprise seule, notamment petite ou moyenne entreprise (PME) et très petite entreprise (TPE), dont les moyens humains et financiers sont contraints, d'identifier la démarche à suivre et de définir une nouvelle stratégie tout en ayant conscience des risques encourus. L'environnement et la typologie de production (make to order, make to stock, mass customisation, etc.) impacteront directement le choix de cette stratégie. Dès lors, il est nécessaire d'accompagner les entreprises, de donner aux acteurs une méthode et des outils permettant de diagnostiquer leur niveau de maturité du 4.0, de définir leurs objectifs et priorités dans leur transformation 4.0 et plus particulièrement sur leur vision de leur chaîne logistique. A ce jour, il n'existe pas de méthode de transformation organisationnelle pour aider les entreprises à se positionner et définir une stratégie leur permettant de prioriser la transformation de leur chaîne logistique vers le 4.0. Les diagnostics pratiqués actuellement reposent essentiellement sur des interviews basées sur l'expérience des " auditeurs » sans s'appuyer sur une méthodologie structurante et des résultats factuels. En partant d'un état de l'art, qui sera détaillé dans la section suivante, nous nous sommes aperçus d'une absence de modèle de transformation 4.0 structurant pour les entreprises et pour leur chaîne logistique. Ce constat nous a amené à faire l'hypothèse que pour faire progresser une chaîne logistique dans son ensemble, il est nécessaire que chaque entreprise constitutive de cette chaîne logistique puisse se projeter à travers des étapes réalistes en se fixant des objectifs atteignables au regard de ses capacités à évoluer dans le domaine du 4.0.

En menant une recherche hypothético-déductive, l'objectif de cet article est de proposer une méthode et des outils permettant aux entreprises participantes à une chaîne logistique donnée de réussir leur transition vers "I'Industrie du futur ». Pour cela, nous devons être en mesure d'évaluer le niveau de performance d'une entreprise par rapport à un objectif global de la chaîne logistique dont elle fait partie et pouvoir l'aider à s'étalonner.

La méthodologie de recherche mise en œuvre dans le cadre de cet article est la suivante : (1) réaliser un état de l'art afin de cerner les enjeux et la problématique actuelle des entreprises pour la transformation 4.0 ; (2) répertorier les principaux domaines de la chaîne logistique (ainsi que leurs axes) où cette transformation devrait avoir lieu afin d'optimiser la chaîne ; (3) identifier les modèles d'évaluation de maturité existante dans la littérature ; (4) proposer un outil et une méthodologie d'évaluation pour aider les entreprises de la chaîne logistique à s'auto évaluer et à définir des priorités dans leur stratégie de transitions vers le 4.0 en conformité avec la chaîne logistique dont elles font partie ; et (5) mettre en œuvre la méthodologie (études de cas avec des industriels).

L'article est structuré de la manière suivante : dans un premier temps, nous présentons les différents domaines de la transformation 4.0 dans les entreprises industrielles ainsi que le modèle d'évaluation de la maturité existants dans la littérature. Puis nous proposons un modèle d'évaluation de la performance de l'entreprise pour les accompagner dans la transformation vers le 4.0 en conformité avec les objectifs de la chaîne logistique dont elles font partie. 
Ce modèle permet à la fois d'évaluer le niveau de maturité actuel de chaque entreprise dans différents axes de développement possible, mais aussi de se comparer et d'avoir une vision globale de leur positionnement par rapport à leurs chaînes logistiques. Cela permet ainsi de visualiser l'écart entre le niveau de maturité actuel d'une entreprise et le niveau de maturité exigée par la chaîne logistique globale donc par le marché. L'analyse de cet écart permet de définir la stratégie de transformation 4.0 à mettre en œuvre dans chaque entreprise considérée. Dans la section "Cas d'étude ", les cas présentés ont pour but de mettre en évidence la mise en application de la méthodologie, la manière dont celle-ci permet d'exploiter les résultats afin de faciliter la prise de décision. Elle permet également de tracer les perspectives et les orientations stratégiques pour les entreprises de la chaîne logistique.

\section{Etude de la littérature : domaines stratégiques de la transition $\mathbf{4 . 0}$}

Cette section présente une étude de la littérature concernant les domaines stratégiques de la transition 4.0 et les modèles d'évaluation de maturité utilisés. Le niveau de maturité désigne la situation (étape) dans laquelle se trouve une organisation par rapport au déploiement de ses processus à partir d'un objectif (Chrissis, Konrad, et Shrum 2008).

De nombreuses recherches se sont intéressées aux aspects technologiques de l'entreprise 4.0 (Vaidya, Ambad, et Bhosle 2018) et de ses implications dans la chaîne logistique (Hermann, Pentek, et Otto 2016 ; Barreto, Amaral, et Pereira 2017 ; Tjahjono et al. 2017). Les aspects technologiques concernent principalement le concept de "smart factory ", c'est-à-dire la création d'un environnement intelligent et apprenant dans le système de production d'une entreprise (Faller et Feldmüller 2015 ; Prinz et al. 2016). Poussé par l'Internet, le monde réel et virtuel de l'entreprise se transforme en "Internet of Things " pour être plus rapide, plus efficient et plus flexible en terme de production, en utilisant des "big data intelligentes". La production est mise en relation avec les systèmes $d$ 'information et de communication les plus modernes pour créer une chaîne logistique intelligente, digitalement connectée et dont la production est organisée en grande partie par elle-même, tout en se basant sur les concepts du Lean (Sanders, Elangeswaran, et Wulfsberg 2016).

Cela suppose également la mise en œuvre de systèmes cyber-physiques, ayant une interconnexion entre les produits, les machines, l'écosystème et même les consommateurs, et ceci à chaque étape de la vie du produit depuis sa conception jusqu'à sa fin de vie (Lee, Bagheri, et Kao 2015 ; Herberer, Lau, et Behrendt 2017).
D'après l'étude allemande de Lichtblau et Stich (2015), seulement $16,8 \%$ des entreprises disposent même d'un système d'évaluation de leurs performances par rapport au 4.0, mais moins de la moitié d'entre elles considèrent que leur système d'indicateurs est adéquat. Ils observent une tendance basée sur la taille de l'entreprise : plus l'entreprise est grande, plus il est probable qu'elle utilise un système d'indicateurs pour mesurer le statut de mise en œuvre de l'industrie 4.0.

\section{Niveaux de maturité}

Le premier modèle d'évaluation du niveau de maturité est CMMI (capability maturity model integration) développé à partir de 2001 par le Software Engineering Institute (SEI), essentiellement pour les entreprises d'informatique. Pour le SEl, la maturité d'un processus est à situer sur une échelle à six niveaux. CMII décrit 25 processus (process areas). Un processus peut être incomplet, exécuté, géré, défini ou documenté, géré quantitativement et optimisé. Une échelle a également été établie pour situer la maturité d'une organisation sur cinq niveaux : initial, discipliné, ajusté, géré quantitativement, optimisé constamment.

En plus de ce modèle générique nous avons identifié dans la littérature plusieurs études concernant l'évaluation de la performance d'une entreprise par rapport à I'Industrie 4.0. Dans la même ligne que CMMI, Lichtblau et Stich (2015) propose un modèle " 4.0 Readiness Model » ayant six niveaux de maturité nommés " outsider », «beginner » pour les newcomers « intermediate »; « experienced» pour les learners ; « expert », « top performer » pour les leaders. Ce modèle considère six dimensions : stratégie et organisation, usine intelligente, opérations intelligentes, produits intelligents, services basés sur les données, employés. Plus récemment Herberer, Lau, et Behrendt (2017) ont proposé un modèle d'évaluation d'un index de maturité sur cinq niveaux : standard, big data, smart data, dark factory, industrial ecosystem. Ils considèrent cinq secteurs de l'entreprise : business management, development, manufacturing, supply chain et service.

Sur la base de ces travaux, nous proposons des niveaux de maturité adaptés à notre problème et permettant l'évaluation des entreprises.

\section{Domaines stratégiques de la transition 4.0}

Les chaînes logistiques sont de plus en plus complexes et difficiles à appréhender. L'enjeu est d'autant plus important que pour que la chaîne logistique globale fonctionne, il est nécessaire que les entreprises partenaires réalisent également une transition vers le 4.0 . 
Plusieurs auteurs discutent de l'impact de l'industrie 4.0 dans la chaîne logistique en montrant la nécessité d'avoir une vision globale. Ils en font ressortir différents domaines d'action. Ainsi Barreto, Amaral, et Pereira (2017) discute de quelques implications technologiques dans la planification, le stockage, le transport et la sécurité des données. Hoffman et Rüsch (2017) identifient des opportunités en termes de décentralisation, autorégulation et efficacité et des implications dans le contexte de juste à temps. Tjahjono et al. (2017) considèrent quatre domaines interconnectés : achats, entreposage, transport et ventes. L'évaluation est basée uniquement sur des critères technologiques. Blanchet et Bergerried (2014) répartissent le concept de l'Industrie 4.0 en six axes - la conception du produit/processus, pilotage/contrôles, opération de fabrication, services (maintenance, intégration), big data et organisation du travail - qui couvrent les principaux aspects du 4.0. Hermann et al. (2016) identifient dans la littérature quatre composantes clés de l'industrie 4.0 : systèmes cyber-physiques, internet des objets, l'internet des services, usines intelligentes. Ils définissent six principes que les entreprises devraient prendre en compte pour implémenter des solutions 4.0 : interopérabilité, virtualisation, décentralisation, capacité de travail en temps réel, orientation services, modularité. Un modèle plus complexe, en trois dimensions (3D), est le modèle RAMI 4.0 (Reference Architectural Model Industrie 4.0) présenté par Hankel et Rexroth (2015). Ce modèle utilise six strates (business, fonctionnel, informationnel, communication, intégration, les actifs) qu'il décline en fonction du cycle de vie du produit et des niveaux hiérarchiques.

En plus des aspects purement technologiques, Schumacher, Erol, et Sihn (2016) propose un modèle de maturité qui intègre également les aspects liés à l'humain et à la culture de l'entreprise que nous souhaitons intégrer également dans notre modèle. D'autant plus que Staun (2015) parle de cinq mutations qui ont lieu quasiment simultanément aujourd'hui :

- technologique : internet de la communication, internet des objets, impressions 3D et internet de l'énergie ;

- conceptuelle (silencieuse) commencé il y a plus d'un siècle, qui change la vision du monde par des découvertes réalisées dans les sciences fondamentales ;

- sociétale avec le passage de l'avoir à l'être initié par groupe d'individus créatifs ;

- économique basée sur la mise en place d'une dimension éthique au cœur de l'économie du marché.

Sur la base ces derniers travaux évoqués, il nous semble important d'intégrer les aspects sociaux et sociétaux ainsi que ceux liés au développement durable et à l'innovation. Malgré l'accès à une base d'articles importante, nous n'avons pas identifié dans la littérature un modèle détaillé prenant en compte tous les aspects sous cités.

L'objectif de notre article est de construire une méthode et un modèle réaliste, exploitable, compréhensible et atteignable dans une transformation vers la chaîne logistique 4.0. Nous partons de l'hypothèse que la transformation vers le 4.0 n'est pas seulement technologique mais concerne l'organisation dans son ensemble. Le passage vers la chaîne logistique 4.0 suppose une transformation globale qui se déploie à plusieurs niveaux : énergétique, écologique, numérique, organisationnel et sociétale (Staun 2015).

Suite à l'étude de la littérature nous avons identifié quatre grands domaines qui représentent au mieux notre vision globale de l'entreprise et les enjeux majeurs qu'elle doit être capable d'intégrer, en s'appuyant sur la chaîne de valeur, afin d'engager une transformation vers le 4.0 :

- la conception des produits et process (Blanchet et Bergerried 2014 ; Hankel et Rexroth 2015 ; Hermann et al. 2016 ; Lee, Bagheri, et Kao 2015 ; Schumacher, Erol, et Sihn 2016 ; Vaidya, Ambad, et Bhosle 2018) ;

- la production, logistique et maintenance (Hankel et Rexroth 2015 ; Hofmann et Rüsch 2017 ; Tjahjono et al. 2017 ; Vaidya, Ambad, et Bhosle 2018) ;

- l'organisation stratégique et innovante (Kagermann, Wahlster, et Helbig 2013 ; Blanchet et Bergerried 2014 ; Faller et Feldmüller 2015 ; Hankel et Rexroth 2015 ; Staun 2015 ; Prinz et al. 2016 ; Schumacher, Erol, et Sihn 2016 ; Hofmann et Rüsch 2017) ;

- I'organisation durable et responsable (Elkington 1998 ; Buhr 2015).

Cette section nous a permis d'identifier les principaux domaines dans lequel les entreprises devront orienter leurs efforts pour une transformation 4.0 réussie et une participation efficiente dans la chaîne logistique globale.

En partant de ces quatre domaines d'analyse, nous proposons par la suite une méthodologie et un modèle de diagnostic détaillé, avec des axes de développement et des niveaux de maturité précis. Une application concrète de cette méthodologie qui s'appuie sur une étude de cas sera également présentée.

\section{Méthodologie de définition d'une stratégie de transformation 4.0}

Afin d'ancrer notre méthodologie dans une démarche globale d'amélioration continue, nous proposons de la structurer suivant les étapes composant la roue de 
Deming (plan - do - check - act ; Johnson 2002). Cette méthodologie, développée par W.E. Deming, offre un cadre de référence et est applicable à l'entreprise entière pour améliorer voire innover de manière contrôlée en minimisant les risques. L'idée étant qu'après un ou plusieurs cycles de la roue de Deming, le niveau attendu dans la transformation 4.0, même si évolutif, soit atteint.

Notre méthodologie intègre, grâce aux outils proposés, une évaluation qualitative du niveau de maturité par rapport aux différents domaines et axes de la transformation 4.0 d'une chaîne logistique (supply chain, SC), une analyse des écarts et une définition de plans d'actions pour la transformation vers le 4.0 (Figure 1).

Les finalités de ces différentes étapes au regard de la transition sont détaillées ci-dessous.

Plan : Définir les objectifs prioritaires du responsable de la chaîne logistique en lien avec la stratégie de l'entreprise (optimal global). Cette phase permet de cadrer le projet, d'identifier l'ensemble des exigences et attendus pour le développement futur de l'entreprise et la chaîne logistique.

Do : Proposer et mettre en œuvre un outil de diagnostic 4.0 de la chaîne logistique et de ses maillons. Cela consiste, par axe, en :

- la définition d'une échelle de maturité et de son niveau de maitrise ;

- I'identification des domaines à prendre compte pour l'évaluation ;

- la définition pour chaque domaine identifié des axes d'évaluation ;

- la réalisation d'un questionnaire à choix multiples à destination des entreprises.

Check : Suite aux réponses au questionnaire, positionner l'entreprise sur chaque axe de chaque domaine de la transformation 4.0 en identifiant les écarts entre la situation actuelle et la situation visée. Les enjeux concernent :

- I'évaluation de chaque maillon de la chaîne logistique ;

- l'évaluation globale de la chaîne logistique ; l'identification et l'analyse des écarts.
Act : Définir un plan d'actions priorisé (long, moyen et court terme) pour l'ensemble de la chaîne logistique ainsi que pour chacun de ses maillons en lien avec la stratégie 4.0.

Dans la suite de notre article nous allons nous focaliser sur les étapes do et check afin de proposer un outil de diagnostic 4.0 qui permettra d'identifier et analyser les écarts par rapport aux objectifs de maturité de chaque axe de transformation 4.0.

\section{Modèle et outil de diagnostic du niveau de maturité $\mathbf{4 . 0}$}

En partant de modèles de la littérature, nous proposons un modèle et un outil de diagnostic mettant en parallèle les enjeux majeurs de la transformation 4.0 ainsi que les différents niveaux de maturité de la chaîne logistique. Dans notre modèle, nous prenons en compte la gestion des données intégrées dans des progiciels ERP/MES (Entreprise Ressources Planning / Manufacturing Execution System), la vision client $360^{\circ}$ et le PLM (product lifecycle management) ainsi que les aspects liés à I'humain et à son environnement (créativité, innovation, chaîne logistique responsable et durable).

\section{Modèle de diagnostic 4.0}

En partant des domaines identifiés dans la section " domaines stratégiques de la transition 4.0 » et des axes définis par l'Alliance industrie du futur (2015) dans le "Guide pratique de l'usine du futur ", nous développons un modèle global réunissant tous les domaines de la transformation 4.0 précédemment présentés. Ce modèle permet de sélectionner les axes d'amélioration et les stratégies à adopter afin que la chaîne logistique puisse répondre aux exigences du donneur d'ordre et aux besoins du client final.

Chaque domaine est détaillé en plusieurs axes dans le modèle de diagnostique 4.0 (Figure 2). Par exemple concernant le domaine « organisation stratégique » les axes sont les suivants :

- Organisation opérationnelle avec : formation et management de ressources ;

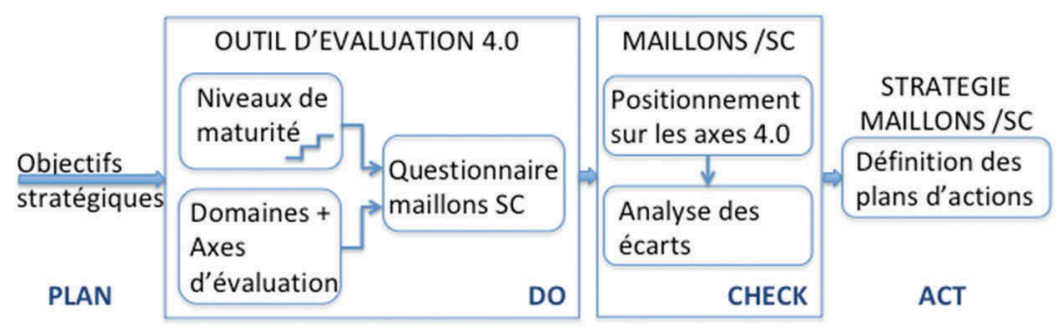

Figure 1. Méthodologie de définition d'une stratégie de transformation 4.0. 


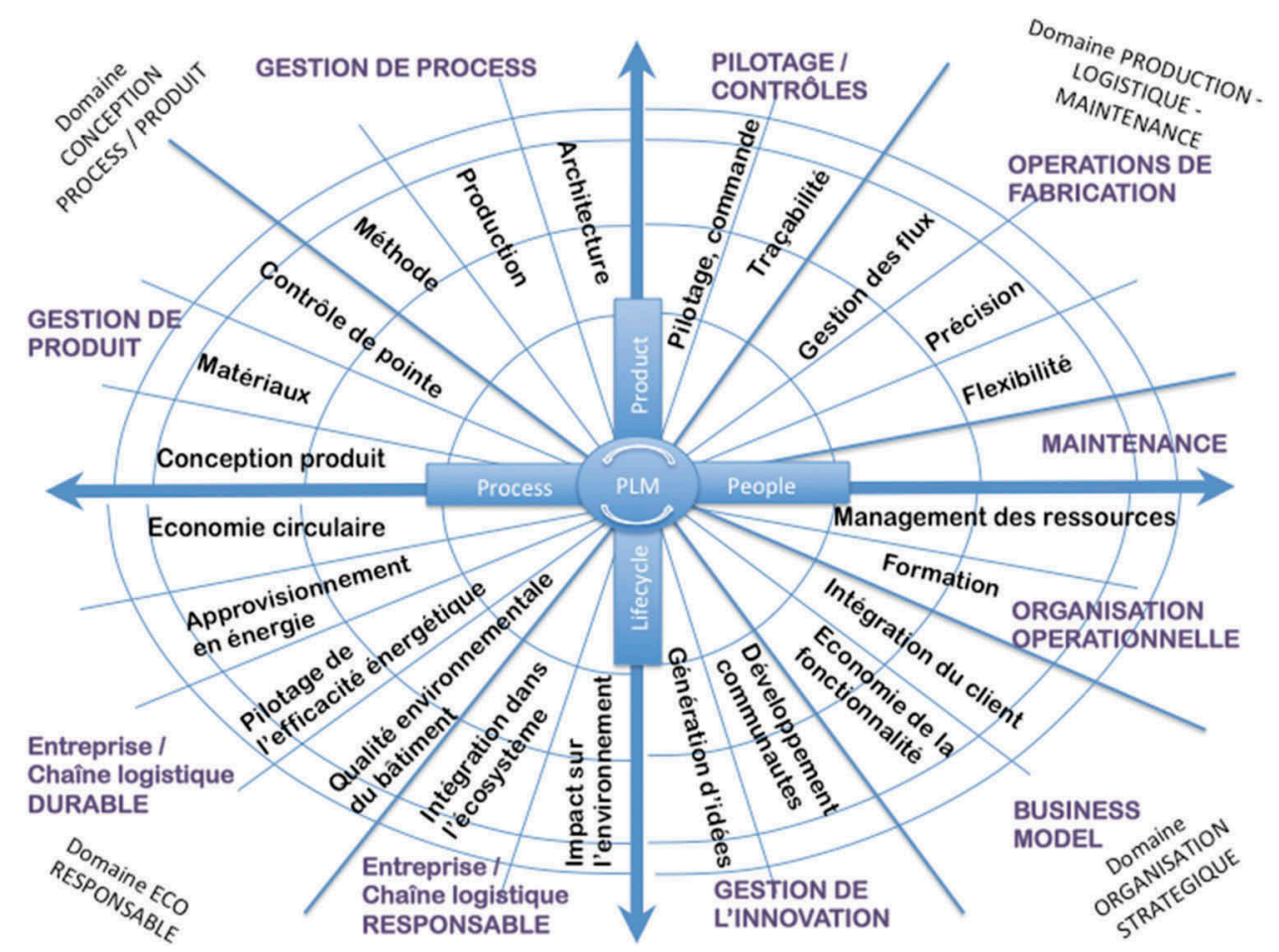

Figure 2. Modèle de diagnostic 4.0 (voir détails en Annexe 1 et 2). PLM : product life-cycle management.

- Business model avec : intégration du client et économie de la fonctionnalité ;

- Gestion de l'innovation avec : développement des communautés et génération des idées.

Pour les phases plan et do de notre méthodologie, en partant des modèles de maturité existants dans la littérature, nous proposons par l'intermédiaire des réponses proposées (questionnaire à choix multiples, QCM) quatre niveaux de maturité et six niveaux de maitrise de la situation actuelle pour le modèle de diagnostic : (1) basique et (2) partiel pour les nouveaux venus qui commencent à s'interroger ; (3) géré et (4) maîtrisé pour les entreprises apprenantes (Prinz et al. 2016), qui ont engagé déjà une transformation vers le 4.0 ; (5) optimisé et (6) innovant pour les leaders qui représentent la vitrine de l'entreprise du futur. Ces niveaux peuvent être apparentés à « outsider » et " basique », " discipliné » et « ajusté », " expert optimisé » et " expert innovant ». Ces derniers ont déjà réussi à atteindre les exigences requises sur tous les aspects de la transformation 4.0 et sont engagés dans une démarche d'innovation continue.

\section{Le questionnaire à choix multiples}

Caractérisation de la situation initiale (actuelle) : le modèle de diagnostic présenté est accompagné d'un questionnaire relatif à chaque domaine et axe identifié. Le Tableau 1 illustre sur un domaine particulier les différentes questions qui se posent aux décideurs des entreprises sur chaque axe de ce domaine. Pour chacune des questions, plusieurs réponses sont possibles afin de caractériser le niveau de maturité. Dans le cadre de cet exemple, le Tableau 2 permet de prendre connaissance de la liste de choix de niveaux de maturité possibles. A chaque question, un choix de quatre réponses est proposé afin de définir non seulement un niveau de maturité actuel mais également d'identifier des solutions possibles à mettre en œuvre. Des commentaires pour chaque choix/niveau facilitent le positionnement. Associé à ces réponses, un niveau de maitrise (de 1 à 6) de la situation actuelle (initiale) de l'entreprise est demandé permettant d'identifier les risques liés à la variabilité de l'existant.

Caractérisation de la situation recherchée (future) : la démarche est identique à la démarche présentée pour la situation initiale. L'élément de différenciation est le niveau de maîtrise de la situation actuelle qui est remplacé par le niveau de priorité pour l'entreprise en fonction de sa stratégie et des ressources à disposition (quatre niveaux de priorités : très forte, forte, faible et très faible ; Tableau 3).

\section{Outil d'aide à la décision stratégique}

Pour l'étape " check » de notre méthodologie nous proposons un outil d'aide à la décision (Tableau 4) qui permet de déterminer le niveau de maturité de chaque maillon puis de l'ensemble de la chaîne logistique sur les différents axes présentés auparavant. II permet également de visualiser le niveau de maturité actuel pour chaque axe ainsi que l'objectif attendu. 


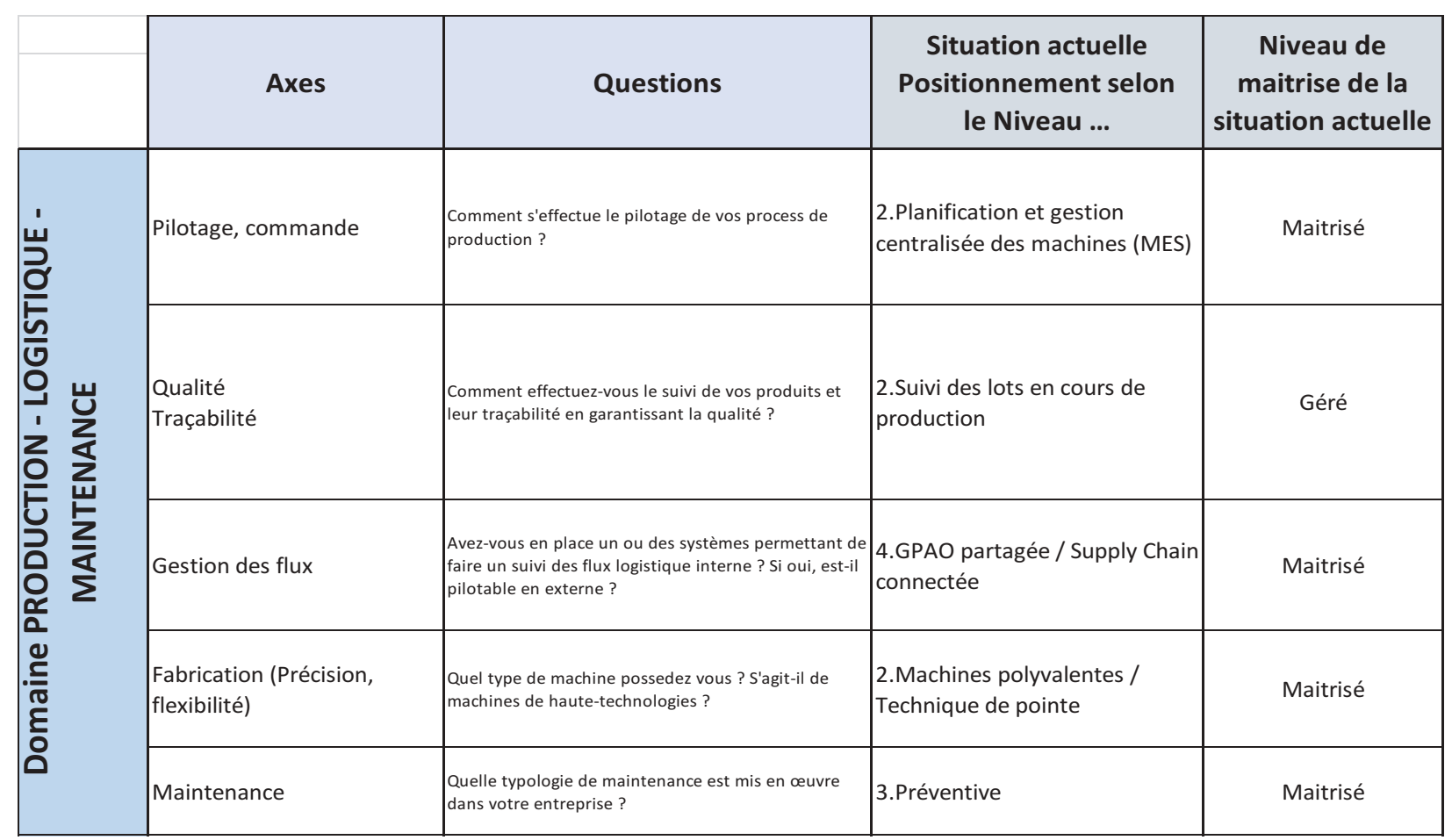

Tableau 1. Exemple de questions/stratégies sur le domaine production-logistique-maintenance. GPAO : gestion de la production assistée par ordinateur.

\begin{tabular}{|l|l|l|l|}
\hline \multicolumn{2}{|c|}{ Questions } \\
\hline
\end{tabular}

Tableau 2. Exemple de choix multiples pour caractériser le niveau de maturité dans le domaine production-logistiquemaintenance.

Suite aux réponses du questionnaire le tableau de l'outil est renseigné et le niveau de maturité pour chaque entreprise est calculé pour chaque axe. Le niveau de maturité global pour la chaîne logistique est automatiquement calculé par la suite. Chaque résultat est comparé avec le niveau de maturité cible que ce soit pour une entreprise ou pour l'ensemble de la chaîne logistique. Les écarts constatés (Figure 3) sont représentés automatiquement sur chaque axe du « Modèle de diagnostique 4.0 » présenté en Annexe 1.

Cette représentation permet de constater rapidement:

- la situation initiale (courbe intérieure - bleu) ;
- la situation visée (courbe extérieure - rouge) ;

- les écarts entre la situation initiale et la situation visée et donc les axes de développement et le plan d'actions associé.

Un calcul quantitatif (équation 1) permet d'évaluer le niveau de maturité $M i$ d'une entreprise $i$ dans sa SC. II permet de déterminer l'influence globale de celle-ci dans sa SC.

II prend en compte le niveau de maturité de l'entreprise $i$ sur l'axe $j(\mathrm{Mij})$ et le niveau de maturité sur l'axe $j$ visé par la SC à laquelle elle appartient $\left(M_{S C i j}\right)$ ainsi que le gap entre les deux (Gapj; équation 2). 


\begin{tabular}{|c|c|c|c|c|}
\hline & Axes & Questions & $\begin{array}{c}\text { Stratégie : Cible - } \\
\text { Positionnement selon le } \\
\text { Niveau (>= actuel) }\end{array}$ & $\begin{array}{c}\text { Cible - Priorité } \\
\text { pour l'entreprise } \\
\text {... }\end{array}$ \\
\hline \multirow{5}{*}{ 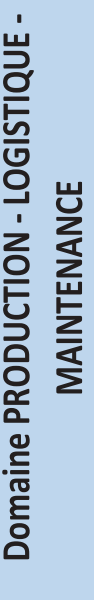 } & Pilotage, commande & $\begin{array}{l}\text { Comment s'effectue le pilotage de vos process de } \\
\text { production? }\end{array}$ & 4.Pilotage à distance & Forte \\
\hline & $\begin{array}{l}\text { Qualité } \\
\text { Traçabilité }\end{array}$ & $\begin{array}{l}\text { Comment effectuez-vous le suivi de vos produits et } \\
\text { leur traçabilité en garantissant la qualité? }\end{array}$ & $\begin{array}{l}\text { 3.Suivi par pièces (Capteur de } \\
\text { condition) et pilotage de la } \\
\text { qualité }\end{array}$ & Forte \\
\hline & Gestion des flux & $\begin{array}{l}\text { Avez-vous en place un ou des systèmes permettant de } \\
\text { faire un suivi des flux logistique interne? Si oui, est-il } \\
\text { pilotable en externe? }\end{array}$ & $\begin{array}{l}\text { 4.GPAO partagée / Supply Chain } \\
\text { connectée }\end{array}$ & Très forte \\
\hline & $\begin{array}{l}\text { Fabrication (Précision, } \\
\text { flexibilité) }\end{array}$ & $\begin{array}{l}\text { Quel type de machine possedez vous ? S'agit-il de } \\
\text { machines de haute-technologies? }\end{array}$ & 4. Robot autonome et intelligent & Très forte \\
\hline & Maintenance & $\begin{array}{l}\text { Quelle typologie de maintenance est mis en œuvre } \\
\text { dans votre entreprise? }\end{array}$ & 4.Prédictive/Prévisionnelle & Forte \\
\hline
\end{tabular}

Tableau 3. Exemple de stratégie cible et priorité sur le domaine production-logistique-maintenance.

$$
M i=\frac{\sum_{j=1}^{m} G a p_{j} * p j}{m}
$$

Avec :

$\mathrm{p}_{j}$ : la pondération de chaque axe $\mathrm{j}$ représentant l'importance de celui-ci dans la politique de l'entreprise ;

$m$ : le nombre d'axes $j$ considérées.

$$
\mathrm{Gap}_{j}=(\mathrm{Mij}-\mathrm{MSC} i j)
$$

Une performance au-delà des attentes ne pouvant pas compenser des lacunes sur un autre axe, si le niveau est atteint ou dépassé on reste sur 0 car atteint $(0$ devient le max) $\operatorname{Gap}_{j}=0$.

Suite à l'analyse des écarts, notre méthodologie prévoit dans l'étape " act » de définir une stratégie et des plans d'actions pour la transformation future des entreprises et de leurs chaînes logistiques vers le 4.0. Pour les entreprises impliquées dans la chaîne logistique, cette stratégie prend en compte une compétitivité durable, les contraintes environnementales et le besoin de création de richesse en minimisant la consommation de ressources. La stratégie globale de la chaîne logistique sera déployée au niveau de chacune des entreprises participantes afin de définir : Quelle est sa stratégie de développement futur ? Quels sont les plans d'actions et solutions à mettre en œuvre ? Quelles sont les priorités ? Quels moyens mettre en œuvre?

\section{Cas d'étude}

Le modèle et l'outil de diagnostic du niveau de maturité 4.0 ont été testés dans plusieurs entreprises implantées en Alsace.
Les entreprises consultées ont les caractéristiques génériques suivantes :

- Industries manufacturières - activités de conception, production et assemblage ;

- Entreprises multi sites ;

- Marché international - clients automobile, aéronautique et industries au sens large de tous secteurs d'activités ;

- Production à la demande en cycle court ;

- Forte personnalisation des produits et grande variabilité ;

- Nombre de salariés compris entre 30 à 2000 (mode : 500 salariés).

Un exemple de résultats obtenus basés pour cet échantillon d'entreprises implantées en Alsace est présenté en Annexe 2. Ces résultats permettent de comparer la situation initiale de chaque entreprise à la situation visée selon les exigences de la chaîne logistique dont elle fait partie. Ainsi des écarts sont mis en évidence sur tous les axes de développement prévus. Cela permet à ces entreprises de définir des stratégies de transformation et de définir des plans d'actions associés.

En analysant les résultats obtenus (schémas Annexe 2), les écarts les plus significatifs sont associés au domaine PLM avec des objectifs visés ambitieux mais en totale adéquation avec les enjeux de l'Industrie du futur. Les priorités stratégiques des entreprises dans le cadre de cette transition concernent également ce même domaine et particulièrement les axes " gestion des flux », " fabrication (précision et flexibilité) » et « maintenance ». 


\begin{tabular}{|c|c|c|c|}
\hline Domaines & Axes & $\begin{array}{c}\text { Niveau de } \\
\text { maturité } \\
\text { actuel / } 4\end{array}$ & $\begin{array}{l}\text { Niveau de } \\
\text { Maitrise / } 6\end{array}$ \\
\hline \multirow{5}{*}{ 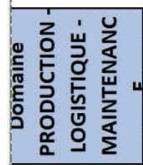 } & Pilotage, commande & 2 & 4 \\
\hline & Qualité, Traçabilité & 2 & 3 \\
\hline & 4 Gestion des flux & 4 & 4 \\
\hline & Fabrication (Précision, flexibilité) & 2 & 4 \\
\hline & Maintenance & 3 & 4 \\
\hline \multirow{5}{*}{ 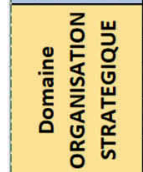 } & Management des ressources & 3 & 4 \\
\hline & Formation & 2 & 4 \\
\hline & Intégration du client & 4 & 6 \\
\hline & Gestion propriété industrielle & 1 & 4 \\
\hline & Génération d'idées & 2 & 5 \\
\hline \multirow{4}{*}{ 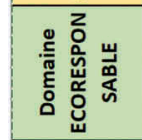 } & Impact sur l'envirronement & 2 & 4 \\
\hline & Qualité environnemental du batiment & 1 & 3 \\
\hline & Approvisionnement en énérgie & 2 & 2 \\
\hline & Économie circulaire & 4 & 4 \\
\hline \multirow{4}{*}{ 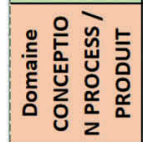 } & Conception produit & 4 & 6 \\
\hline & Matériaux & 1 & 1 \\
\hline & Contrôle qualité & 4 & 6 \\
\hline & Production & 2 & 4 \\
\hline Domaines & Axes & $\begin{array}{l}\text { Niveau de } \\
\text { maturité } \\
\text { Cible / } 4\end{array}$ & Priorité \\
\hline \multirow{5}{*}{ 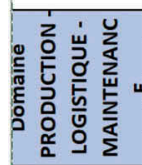 } & Pilotage, commande & 4 & Forte \\
\hline & Qualité, Traçabilité & 3 & Forte \\
\hline & Gestion des flux & 4 & Très forte \\
\hline & Fabrication (Précision, flexibilité) & 4 & Très forte \\
\hline & Maintenance & 4 & Forte \\
\hline \multirow{5}{*}{ 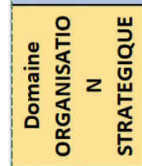 } & Management des ressources & 3 & Forte \\
\hline & Formation & 3 & Forte \\
\hline & Intégration du client & 3 & Très forte \\
\hline & Gestion propriété industrielle & 3 & Forte \\
\hline & Génération d'idées & 3 & Forte \\
\hline \multirow{4}{*}{ 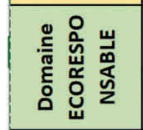 } & Impact sur l'envirronement & 4 & Forte \\
\hline & Qualité environnemental du batiment & 3 & Forte \\
\hline & Approvisionnement en énérgie & 4 & Forte \\
\hline & Économie circulaire & 3 & Très forte \\
\hline \multirow{4}{*}{ 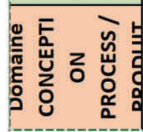 } & Conception produit & 3 & Très forte \\
\hline & Matériaux & 3 & Faible \\
\hline & Contrôle qualité & 2 & Forte \\
\hline & Production & 3 & Très forte \\
\hline
\end{tabular}

Tableau 4. L'outil d'évaluation de la maturité (extrait).

Pour les entreprises qui présentent le niveau de maturité 4.0 le plus élevé, les axes de développement du domaine ecoresponsable (économie circulaire, etc.) sont visés dans cette transition 4.0 et sont fondamentalement liés au management de la chaîne logistique (voir Annexe 2).

L'évaluation quantitative du niveau de maturité, relative aux attentes de la chaîne logistique, est présentée sur le Tableau 5. Elle permet dans un premier temps d'observer une variabilité des résultats avec une influence sur la chaîne logistique significative pour certaines entreprises, notamment les 2, 3, 5, 7 et 8. L'entreprise 4, quant à elle, présente un retard faible par rapport à aux objectif de sa chaîne logistique.

Dans trois entreprises, des axes n'ont pu être évalué. Lorsqu'un axe n'est pas évalué, il n'est pas pris en compte dans le calcul.

Dans trois entreprises, des niveaux de maturité strictement supérieure à l'attente de la chaîne logistique sont observés. Ces trois entreprises présentent pourtant des scores parmi les plus faibles rencontrés indiquant un déséquilibre entre l'effort effectué et celui attendu.

L'aspect management des ressources apparaît comme fondamental pour toutes les entreprises consultées et nous retrouvons la problématique de l'intégration de l'homme dans la transformation 4.0 avec des conséquences sur l'évolution de métiers et des compétences en garantissant une qualité de vie au travail : I'industrie 4.H.

Ces études de cas, réalisées suite aux interviews de managers, nous ont permis de tester nos hypothèses et d'appliquer sur des cas concrets notre méthodologie et notre modèle proposé. Pour les entreprises concernées, cette mise en application a été une véritable aide méthodologique pour identifier le niveau souhaité de leur chaîne logistique dans les différents domaines du 4.0, pour identifier leur niveau de maturité actuel et finalement pour fixer des objectifs stratégiques pertinentes et atteignables. 


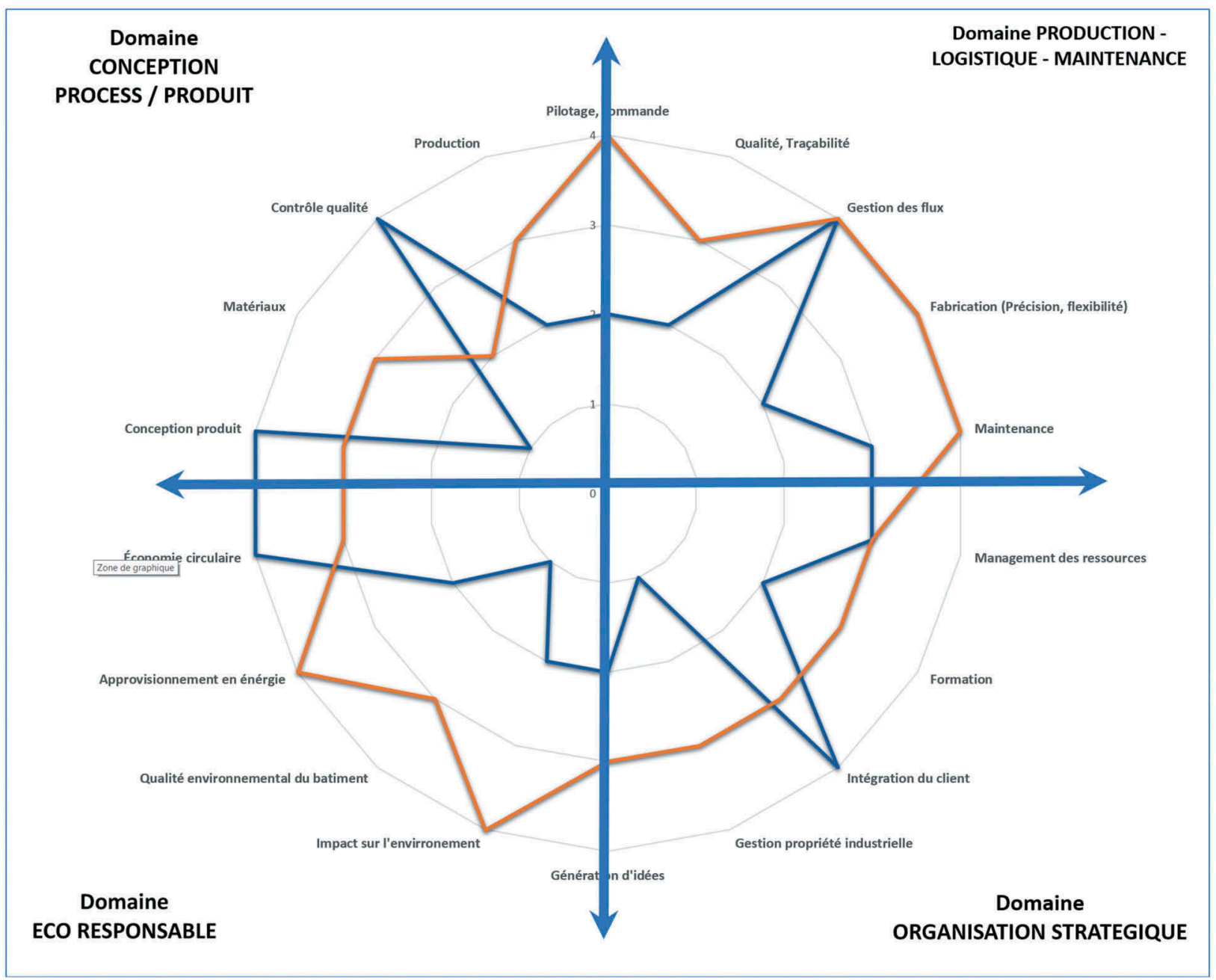

Figure 3. Représentation des écarts.

\begin{tabular}{|c|c|c|c|}
\hline Entreprise & $\mathbf{M i}$ & $\begin{array}{c}\text { Nombre d'axes } \\
\text { non évalués }\end{array}$ & $\begin{array}{c}\text { Nombre d'axes } \\
\text { maturité } \\
\text { supérieure }\end{array}$ \\
\hline 1 & $-1,20$ & 2 & \\
\hline 2 & $-3,11$ & & \\
\hline 3 & $-3,00$ & 9 & \\
\hline 4 & $-0,61$ & & 4 \\
\hline 5 & $-3,33$ & & \\
\hline 6 & $-2,61$ & & \\
\hline 7 & $-3,31$ & 2 & 1 \\
\hline 8 & $-3,00$ & & \\
\hline
\end{tabular}

Tableau 5. Evaluation quantitative du niveau de maturité.

\section{Conclusions et perspectives}

Dans la dynamique du redressement productif de notre tissu industriel français, cet article vise à proposer une méthodologie et un outil de définition de la stratégie de transformation 4.0 pour la chaîne logistique.

Cette contribution managériale aide chaque entreprise de façon personnalisée à concevoir et à piloter une stratégie de transformation vers le 4.0, en priorisant les axes de développement dont l'axe essentiel de la chaîne logistique, pour répondre aux exigences des clients.

Les entreprises pourront ainsi gagner en agilité et efficience sans oublier la rentabilité, tout en étant durables et respectueuses de l'homme et de son environnement. Elles pourront aussi s'insérer plus facilement dans les chaînes logistiques 4.0 (des fournisseurs jusqu'au client final).

Ces contributions permettent également de pouvoir comparer les entreprises par secteur d'activités et typologie, définir des standards éventuels et développer implicitement une démarche de « benchlearning ».

L'outil permet les agrégations de toutes les évaluations et génère les diagrammes automatiquement permettant à l'entreprise d'avoir une visualisation directe de son positionnement par rapport aux objectifs de la chaîne logistique. Elle peut alors construire une stratégie argumentée sur la base des indicateurs de l'entreprise par rapport aux objectifs de la chaîne logistique.

Lors de la mise en œuvre de la méthodologie et de l'outil présenté, les principales difficultés rencontrées ont été les suivantes: 
- Identifier l'interlocuteur ayant suffisamment de recul et de connaissances des processus de l'entreprise pour compléter l'intégralité du questionnaire ;

- La confidentialité des données relatives à l'évaluation de la situation actuelle, les niveaux visés en fonction de la stratégie de l'entreprise avec la priorisation des axes;

- L'élaboration d'un outil compréhensible, facile d'utilisation et adapté à différentes typologies d'entreprises. A chaque retour, une évaluation de l'outil a été réalisée avec pour résultats : " le questionnaire permet un réel positionnement dans le 4.0, les axes ainsi que les questions et niveaux sont pertinents. La méthodologie proposée ne propose malheureusement pas directement d'actions immédiates à mettre en œuvre... ».

Ainsi, en perspectives, nous envisageons de proposer un modèle spécifique de gestion des risques, permettant la prise de décisions dans la sélection d'une stratégie de transition, en partant de la caractérisation du modèle d'influence des risques sur le projet de transformation vers la chaîne logistique 4.0.

II est notamment attendu une cartographie des processus agiles et des plans d'action en fonction de la stratégie choisie par chaque entreprise afin de garantir l'efficience opérationnelle tout en générant de l'innovation.

\section{Déclaration}

Les auteurs confirment qu'il n'y a aucun conflit d'intérêt

\section{Les auteurs}

loana Deniaud, ingénieur en Ingénierie de systèmes de production et docteur en génie industriel, est actuellement maître de conférences à I'Université de Strasbourg et chercheur au laboratoire BETA, UMR 7522 du CNRS. Ses recherches portent sur la gestion de projets innovants à l'aide d'outils et de méthodes d'ingénierie système et d'amélioration continue. Elle a participé à de nombreux projets avec des entreprises industrielles et a publié ses résultats de recherche dans des revues et conférences scientifiques.

François Marmier, ingénieur en génie industriel, est actuellement maître assistant à l'École nationale supérieure des Mines d'Albi-Carmaux et chercheur au Centre de génie industriel. II a obtenu son doctorat en génie industriel à I'Université de Franche-Comté. Ses recherches portent sur l'intégration des aspects humains, l'incertitude et le risque de prendre de meilleures décisions en matière de projets, de logistique et de services. II a participé à divers projets de recherche liés au développement de systèmes de décisions pour la gestion de projets.

Jean-Louis Michalak, professeur agrégé en sciences industrielles de l'ingénieur et ingénierie mécanique, enseigne à I'Université de Strasbourg. II développe tant les enseignements dans les domaines de la gestion industrielle que les relations avec les entreprises, en réalisant de nombreuses actions de conseil/formation. Depuis plusieurs années, il développe une usine ecole "Lean Management » et " démonstrateur de l'industrie 4.0 » permettant à ses étudiants de masters de monter en compétences dans ces domaines. Dans ce cadre, il anime régulièrement des groupes de travail dans le domaine de la transformation numérique des entreprises permettant de construire des projets enseignement/recherche grâce à une forte coopération entre le monde académique et le monde industriel.

\section{ORCID}

loana Deniaud (D) http://orcid.org/0000-0002-8847-5689

François Marmier (D) http://orcid.org/0000-0001-9264-293X

\section{Références}

Alavi, S., D. Abd. Wahab, N. Muhamad, and B. Arbab Shirani. 2014. "Organic Structure and Organisational Learning as the Main Antecedents of Workforce Agility." International Journal of Production Research 52 (21): 6273-6295. doi:10.1080/00207543.2014.919420.

Alliance Industrie du Futur. 2015. Guide pratique de I'Usine du Futur - Enjeux et panorama de solutions. consulté sur : https://www.pfa-auto.fr/

Barreto, L., A. Amaral, and T. Pereira. 2017. "Industry 4.0 Implications in Logistics: An Overview." Procedia Manufacturing 13: 1245-1252. doi:10.1016/j. promfg.2017.09.045.

Blanchet, M., et R. Bergerried. 2014. Industrie 4.0-les leviers de la transformation. Gimélec. consulté sur: http://eduscol. education.fr/sti/ressources_techniques/

Brown, S., and J. Bessant. 2003. "The Manufacturing Strategycapabilities Links in Mass Customisation and Agile Manufacturing-An Exploratory Study." International Journal of Operations \& Production Management 23 (7): 707-730. doi:10.1108/01443570310481522.

Buhr, D. 2015. Social Innovation Policy for Industry 4.0. FriedrichEbert-Stiftung, Division for Social and Economic Policies. consulté sur : http://www.fes-china.org/en/publications/ detail/social-innovation-industry-4.0-en.html

Chrissis, M. B., M. Konrad, et S. Shrum. 2008. CMMI $2^{e}$ Edition Guide des bonnes pratiques pour l'amélioration des processus - CMMI (r) pour le développement, version 1.2. SEI, Pearson Education France. ISBN 9782744073045.

Christopher, M., and D. R. Towill. 2000. "Supply Chain Migration from Lean and Functional to Agile and Customised." Supply Chain Management: An International Journal 5 (4): 206-213.

Duc, C. 2018. La moitié des sociétés procède à des innovations. Insee Première $\mathrm{n}^{\circ} 1709$. consulté sur : https://insee.fr/fr/statistiques/3606601

Elkington, J. 1998. Cannibals with Forks: The Triple Bottom Line of the 21st Century. Gabriola Island, BC: New Society Publishers.

Faller, C., and D. Feldmüller. 2015. "Industry 4.0 Learning Factory for Regional SMEs." Procedia Cirp 32: 88-91. doi:10.1016/j.procir.2015.02.117.

Hankel, M., and B. Rexroth. 2015. The Reference Architectural Model Industrie 4.0 (Rami 4.0). ZVEl, 2, 2. Consulté sur: https://www.plattform-i40.de/ 
Herberer, S., L. K. Lau, and F. Behrendt. 2017. "Developement of an Industrie 4.0 Maturity Index for Smal and Medium-sized Entreprises." IESM Conference, Saarbrucken.

Hermann, M., T. Pentek, and B. Otto. 2016. “Design Principles for Industrie 4.0 Scenarios." IEEE 49th Hawaii International Conference on System Sciences (HICSS) 3928-3937. doi:10.1109/HICSS.2016.488.

Hofmann, E., and M. Rüsch. 2017. "Industry 4.0 And the Current Status as Well as Future Prospects on Logistics." Computers in Industry 89: 23-34. doi:10.1016/j. compind.2017.04.002.

Johnson, C. N. 2002. "The Benefits Fo PDCA." Quality Progress 35 (5): 120.

Kagermann, H., W. Wahlster, and J. Helbig, eds., 2013. "Recommen-dations for Implementing the Strategic Initiative Industrie 4.0." Final report of the Industrie 4.0 Working Group. consulté sur : https://www.din.de

Lee, J., B. Bagheri, and H. A. Kao. 2015. "A Cyber-physical Systems Architecture for Industry 4.0-based Manufacturing Systems." Manufacturing Letters 3: 18-23. doi:10.1016/j.mfglet.2014.12.001.

Lichtblau, K., and V. Stich. 2015. Industrie 4.0-Readiness. Ouvrage collectif, Impuls-Stiftung, Foundation for mechanical engineering, plant engineering and information technology. consulté sur: http://www.iosb.fraunhofer.de

O'Reilly, C. A., III, and M. L. Tushman. 2013. "Organizational Ambidexterity: Past, Present, and Future." Academy of
Management Perspectives 27 (4): 324-338. doi:10.5465/ amp.2013.0025.

Prinz, C., F. Morlock, S. Freith, N. Kreggenfeld, D. Kreimeier, and B. Kuhlenkötter. 2016. "Learning Factory Modules for Smart Factories in Industrie 4.0." Procedia CiRp 54: 113118. doi:10.1016/j.procir.2016.05.105.

Sanders, A., C. Elangeswaran, and J. P. Wulfsberg. 2016. "Industry 4.0 Implies Lean Manufacturing: Research Activities in Industry 4.0 Function as Enablers for Lean Manufacturing." Journal of Industrial Engineering and Management (JIEM) 9 (3): 811-833. doi:10.3926/jiem.1940.

Schumacher, A., S. Erol, and W. Sihn. 2016. "A Maturity Model for Assessing Industry 4.0 Readiness and Maturity of Manufacturing Enterprises." Procedia Cirp 52: 161-166. doi:10.1016/j.procir.2016.07.040.

Staun, J. 2015. Les clés du futur. Réinventer ensemble la société, l'économie et la science. Ed. Plon. ISBN 2259217478.

Tjahjono, B., C. Esplugues, E. Ares, and G. Pelaez. 2017. "What Does Industry 4.0 Mean to Supply Chain?" Procedia Manufacturing 13: 1175-1182. doi:10.1016/j. promfg.2017.09.191.

Vaidya, S., P. Ambad, and S. Bhosle. 2018. "Industry 4.0-A Glimpse." Procedia Manufacturing 20 (1): 233-238. doi:10.1016/j.promfg.2018.02.034.

Womack, J. P., and D. T. Jones. 1997. "Lean Thinking—Banish Waste and Create Wealth in Your Corporation." Journal of the Operational Research Society 48 (11): 1148. doi:10.1057/palgrave.jors.2600967. 


\section{Annexe 1. Modèle de diagnostic 4.0}

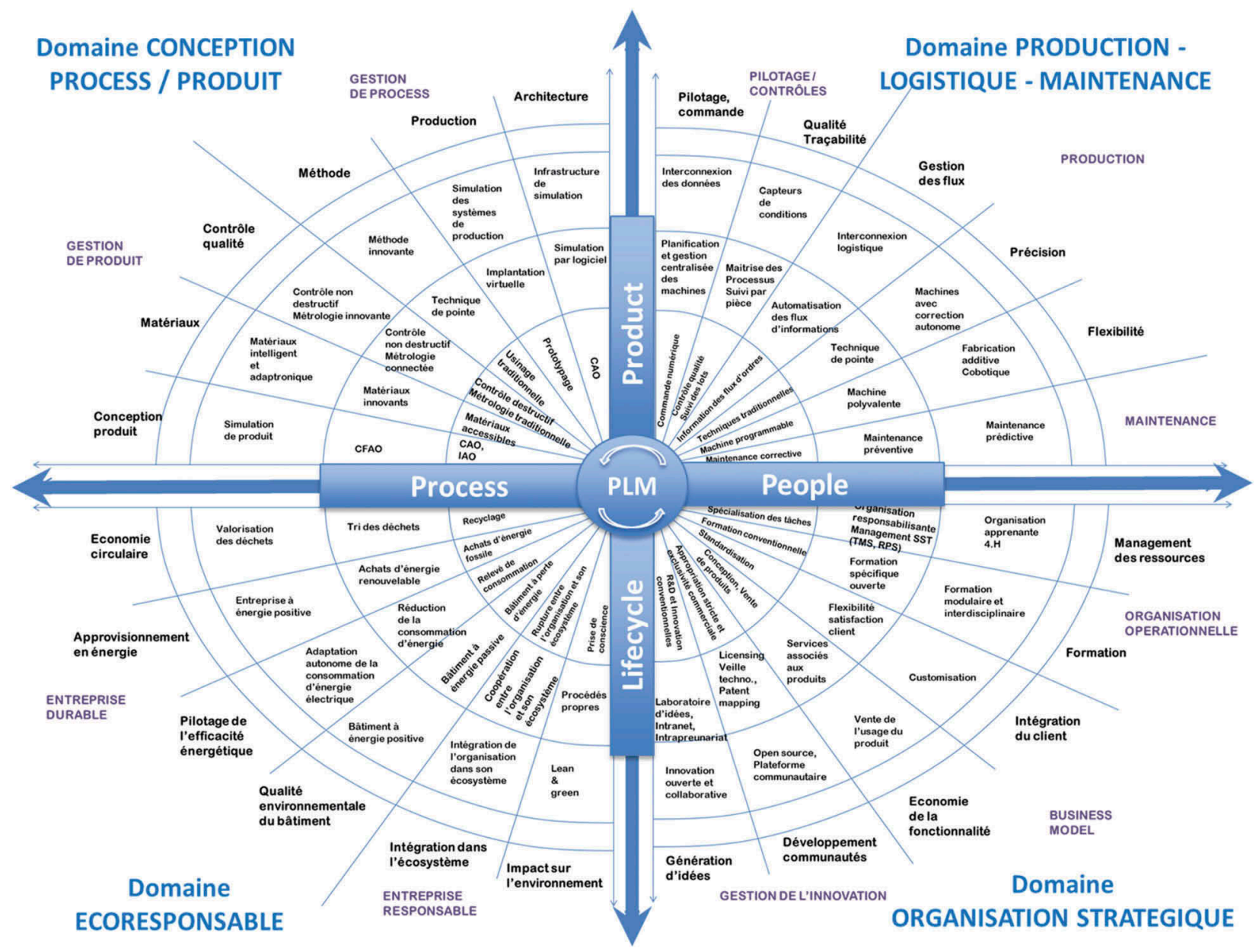


Annexe 2. Exemples de résultats obtenus basés sur un échantillon d'entreprises

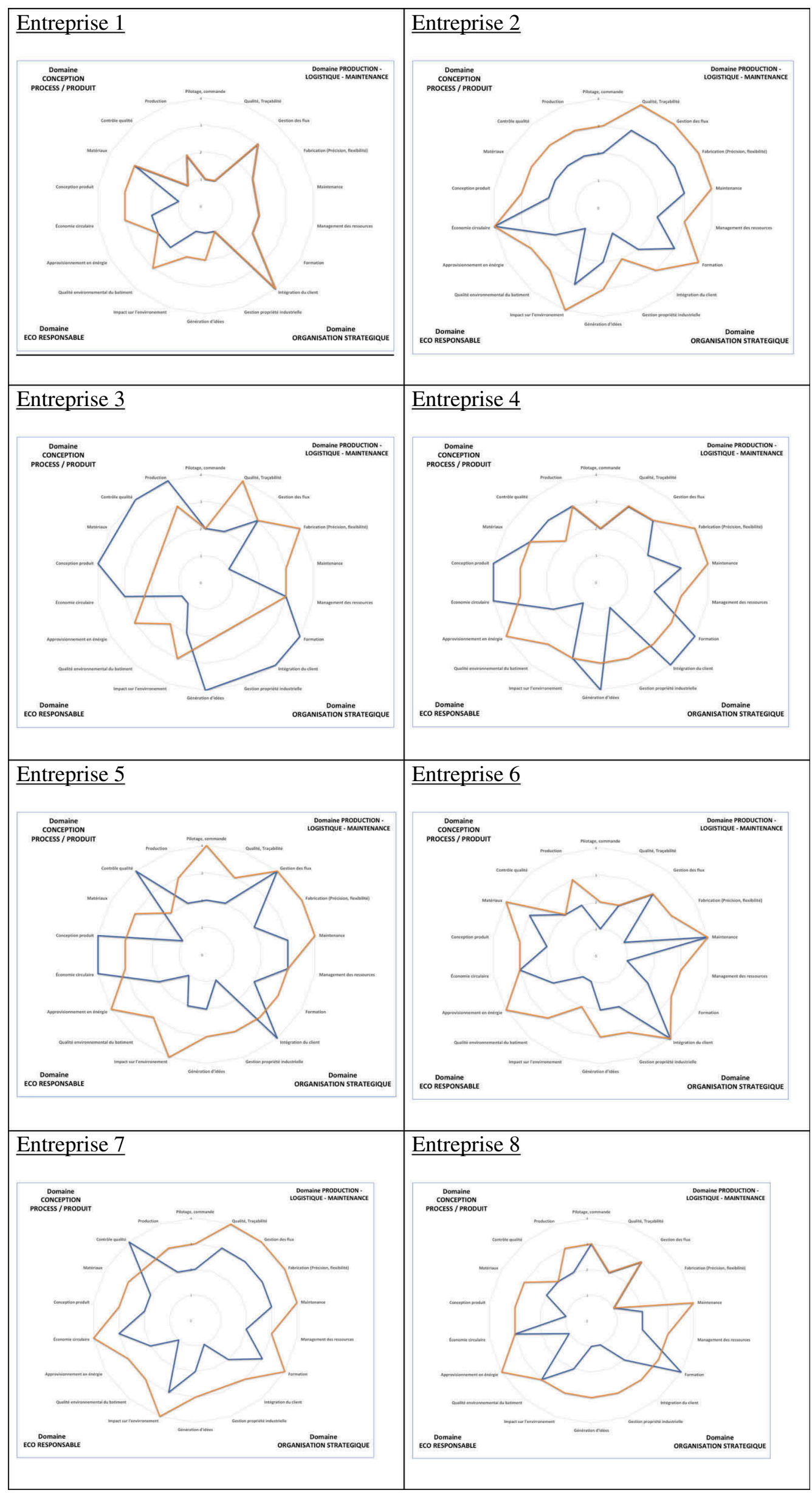

